\title{
Neuronal Substrates for Choice Under Ambiguity, Risk, Gains, and Losses
}

\author{
Kip Smith • John Dickhaut • Kevin McCabe • José V. Pardo \\ Kansas State University, Department of Psychology, Manhattan, Kansas 66506 \\ University of Minnesota, Carlson School of Management, Department of Accounting, \\ Minneapolis, Minnesota 55455 \\ George Mason University, Economics and Law, Krasnow Center, 4400 University Drive, \\ MSN 5C7, Fairfax, Virginia 22030 \\ Veterans Affairs Medical Center, Cognitive Neuroimaging Unit, Minneapolis, Minnesota 55417, \\ and University of Minnesota, Department of Psychiatry, Minneapolis, Minnesota 55455 \\ kip@ksu.edu・jdickhaut@csom.umn.edu・kmccabe@gmu.edu • pardo001@umn.edu
}

\begin{abstract}
$\mathrm{E}$ conomic forces shape the behavior of individuals and institutions. Forces affecting individual behavior are attitudes about payoffs (gains and losses) and beliefs about outcomes (risk and ambiguity). Under risk, the likelihoods of alternative outcomes are fully known. Under ambiguity, these likelihoods are unknown. In our experiment, payoffs and outcomes were manipulated independently during a classical choice task as brain activity was measured with positron emission tomography (PET). Here, we show that attitudes about payoffs and beliefs about the likelihood of outcomes exhibit interaction effects both behaviorally and neurally. Participants are risk averse in gains and risk-seeking in losses; they are ambiguity-seeking in neither gains nor losses. Two neural substrates for choice surfaced in the interaction between attitudes and beliefs: a dorsomedial neocortical system and a ventromedial system. This finding reveals that the brain does not honor a prevalent assumption of economics - the independence of the evaluations of payoffs and outcomes. The demonstration of a relationship between brain activity and observed economic choice attests to the feasibility of a neuroeconomic decision science.
\end{abstract}

\section{Introduction}

A gamble with known payoffs with a well-defined probability distribution is termed "risky" (Ellsberg 1961, Knight 1921). In contrast, a gamble with known payoffs with probabilities that are not well defined is termed "ambiguous." ${ }^{1}$ Decision-making research

\footnotetext{
${ }^{1}$ We characterize ambiguity as lack of knowledge of the distribution of outcomes. Like Ellsberg (1961), we operationalize ambiguity by not telling subjects how many balls of each color are in an urn (different colors pay different amounts if drawn.) Others characterize ambiguity as a probability distribution on the possible color composition of the urn (Howard 1992). Ambiguous settings have been characterized as settings in which the competence of the decision maker is challenged (Heath and Tversky 1991). For a more complete discussion of ambiguity see Camerer and Weber (1992).
}

on choice behavior finds risk-avoiding in gains, riskseeking in losses, and ambiguity-avoiding in gains (Kahneman and Tversky 1979, Curley et al. 1986, Cohen et al. 1987).

To assess the degree of risk-avoiding (seeking), participants were asked to choose between two risky gambles (the risk condition, Figure 1, Row A). The gamble on the left is posed as a container with 30 red, 30 blue, and 30 yellow marbles. The numbers below the arrows indicate that every red and blue marble is worth $\$ 30$ and every yellow marble is worth nothing. The gamble on the right is posed as a container with 30 red, 30 blue, and 30 yellow marbles. Every red marble is worth $\$ 50$, every blue $\$ 6$, and every yellow $\$ 4$. 
Figure 1 Experimental Task Paradigm for Choice Processing Under Ambiguity, Risk, Gains, and Losses

\begin{tabular}{|c|c|c|c|c|c|c|}
\hline \multirow{5}{*}{ A $\begin{array}{l}\text { Risk/Gains } \\
\text { (RG condition) }\end{array}$} & \multicolumn{3}{|c|}{ Gamble 1} & \multicolumn{3}{|c|}{ Gamble 2} \\
\hline & $\mathbf{R}$ & B & $\mathbf{Y}$ & $\mathbf{R}$ & B & $\mathbf{Y}$ \\
\hline & 30 & 30 & 30 & 30 & 30 & 30 \\
\hline & $\uparrow$ & $\uparrow$ & $\uparrow$ & $\uparrow$ & $\uparrow$ & $\uparrow$ \\
\hline & 30 & 30 & 0 & 50 & 6 & 4 \\
\hline \multirow{5}{*}{ B Ambiguity/Gains } & \multicolumn{3}{|c|}{ Gamble 1} & \multicolumn{3}{|c|}{ Gamble 2} \\
\hline & $\mathbf{R}$ & B & $\mathbf{Y}$ & $\mathbf{R}$ & B & $\mathbf{Y}$ \\
\hline & 30 & \multicolumn{2}{|c|}{60} & 30 & 30 & 30 \\
\hline & 个 & $\pi$ & $\kappa$ & $\uparrow$ & $\uparrow$ & $\uparrow$ \\
\hline & 30 & 30 & 0 & 50 & 6 & 4 \\
\hline
\end{tabular}

Note. (A) A sample stimulus for a pair of risky gambles posing gains (condition RG). The groups of squares, numbers, and arrows indicate the distribution of red, blue, and yellow marbles in two containers. The numbers below the arrows signify the payoff in dollars of each of the marbles at the arrowhead. After an initial endorsement of $\$ 190$ cash, the participant's task is to choose the container from which one marble would be drawn. The gamble on the left has the lower payoff variance and is considered to be less risky. (B) A sample stimulus contrasting an ambiguous gamble (on the left) and a risky gamble posing gains (condition AG). The slanted arrows indicate there is a total of 60 blue and yellow marbles in the ambiguous gamble. The precise numbers of blue and yellow are not known. The risky gamble is identical to that shown in Figure $1 \mathrm{~A}$.

The two gambles have the same expected value ${ }^{2}$ but different spreads (variance in payoffs). We use spread as our index of riskiness, with greater spreads reflecting greater risk. ${ }^{3}$ For example, the gamble on the left of Figure 1, Row A, has the smaller spread $(30-0<$ $50-4)$ and is considered less risky. A participant who prefers the gamble with the smaller spread (given a choice between a pair of risky gambles with the same expected value), e.g., the gamble on the left in Figure 1, Row A, is said to avoid risk.

To assess the degree of ambiguity-avoiding (seeking), participants were asked to choose between an ambiguous gamble and a risky gamble (the ambiguity condition, Figure 1, Row B). In the example shown in Figure 1, Row B, the gamble on the left is posed by a container with 30 red marbles, each worth $\$ 30$, and 60 blue and yellow marbles. While each red and

\footnotetext{
${ }^{2}$ Expected value (EV) is defined as the sum of the products of the payoffs and their probabilities. Here the EV of the gamble on the left is $(30 * \$ 30+30 * \$ 30+30 * \$ 0) /(30+30+30)=\$ 20$ and the EV of the gamble on the right is $(30 * \$ 50+30 * \$ 6+30 * \$ 4) /(30+30+30)=$ $\$ 20$.

${ }^{3}$ The assumption that in the range of dollar payoffs subjects are risk averse strictly holds for quadratic utility functions.
}

blue marble is known to be worth $\$ 30$, and each yellow marble is known to be worth nothing, the precise numbers of blue and yellow marbles are not known. There could be as many as 60 blue (yellow) marbles, as few as none, or any number in between (with the numbers of blue and yellow summing to 60). This intrinsic lack of knowledge about the distribution of marbles (payoffs) exemplifies ambiguity. ${ }^{4}$

The risky gamble in the ambiguity condition (on the right side of Figure 1, Row B) is identical to that on the right side of Figure 1, Row A, (the risk condition). This replication allowed us to assess ambiguity avoidance using the assumption of transitivity. For example, a participant who prefers the gamble on the left in the risky condition, but who does not prefer its ambiguous counterpart (like that on the left in Figure 1, Row B), is said to avoid ambiguity.

\footnotetext{
${ }^{4}$ We employ the term ambiguity avoidance as it has typically been studied in experiments. See, e.g., Curley and Yates (1985). Gilboa and Schmiedler (1989) employ a more restrictive definition than we have used here. The operationalization of their definition would mean that our test be augmented by an additional subject comparison in which the blue and yellow balls on the ambiguous gamble are interchanged.
} 
Inspection of Figure 1 reveals that the only differences visually between the risk and ambiguity conditions are the substitution of the 60 for the pair of 30 s, and the presence of diagonal rather than vertical arrows connecting the numbers representing payoffs and their likelihoods. These minimal changes were intended to capture the difference between risk and ambiguity but to avoid wholesale visual contrasts that might generate a confound in the PET images. The full set of stimuli is available as supplementary material from the corresponding author.

In our experiment, for each choice between gambles posing gains (the ambiguity-gains, AG, and riskgains, RG, conditions), there was an equivalent choice between gambles posing losses (conditions $\mathrm{AL}$ and $\mathrm{RL}$ ). The stimuli in the loss conditions were identical to those in Figure 1 with the addition of minus signs $(-)$ in the row of numbers representing payoffs. This manipulation allowed us to distinguish risk-seeking (avoiding) under gains (losses), as well as ambiguityseeking (avoiding) under gains (losses). In the discussion to follow, AG refers to the ambiguous condition posing gains (like that shown in Figure 1, Row B), AL refers to the ambiguous condition posing losses, $\mathrm{RG}$ refers to the risk condition posing gains (like that shown in Figure 1, Row A), and RL refers to the risk condition posing losses.

\section{Methods}

\section{Human Participants}

Nine healthy, right-handed medical students (3 females and 6 males with a mean age of 27 years, s.d. 3 years) participated in the study. The volunteers gave written informed consent according to guidelines of the University of Minnesota Institutional Review Board and the VA Radioactive Drug Research Committee. Medical students were chosen because their familiarity with medical equipment was expected to minimize confounds that might be introduced by the PET imaging environment.

\section{Task Paradigm}

After reading the instructions to participants and engaging in a series of practice trials, a participant received an initial endowment of $\$ 190$ cash. The endowment was held in the left (nondominant) hand while the participant was in the PET scanner. The stimuli and task emulated and extended Ellsberg's paradigm (1961) in which participants had to indicate from which of two containers, each containing a total 90 red, blue, and yellow marbles, they would rather draw a marble at random. They could also indicate lack of preference between containers. Participants saw four task conditions: risk gains (RG, Figure 1, Row A), and risk losses (RL), ambiguity gains (AG, Figure 1, Row B), and ambiguity losses (AL). (There were also several additional conditions, e.g., certainty, resting with eyes closed, that are extraneous for this paper.) Conditions were presented in random order across participants. Each experimental condition consisted of a block of 27 choice pairs each presented in random order. Ambiguous stimuli were on the left of each pair.

After all scans had been conducted, one of the gains trials was chosen at random. The gamble chosen by the participant for that trial during the scan was played with real marbles in a plastic container. Similarly, one loss trial was randomly chosen and the gamble chosen by the participant was played. The participant inspected the container to verify its contents and then held the urn above the experimenter's head while the experimenter selected a marble. The colors of the selected marbles, one from a gains trial and one from a losses trial, determined the participant's additional gain and loss. The participant's total payoff was the initial endowment of $\$ 190$ plus any additional gain, less any loss. On average, participants earned \$193 (s.d. \$20).

Several features of the task paradigm are noteworthy: a randomized block design; an absence of feedback following each trial (and a lack of varying wealth effects); a link between performance and feedback at the end of the study; a possibility for participants to provide a preference or a lack thereof; and the use of the participant's choices in the determination of additional gains and losses.

\section{Imaging}

In addition to classifying observable behavior into risk and ambiguity preferences, we used positron emission tomography (PET) and a tracer $\left(\mathrm{H}_{2}{ }^{15} \mathrm{O}\right)$ to 
estimate regional cerebral blood flow ( $\mathrm{rCBF}$, a standard indicator for brain activity). rCBF was estimated from tissue radioactivity (after correction with measured two-dimensional attenuation) using a Siemens ECAT 953B scanner (Knoxville, Tenn.) with septae retracted, i.e., three-dimensional acquisition (Silbersweig et al. 1993). An arm vein was used for access. The participant's head position was stabilized with a vacuum-molded pillow. A slow-bolus of $\left(\mathrm{H}_{2}{ }^{15} \mathrm{O}\right)$ was injected intravenously $(9.25 \mathrm{Mbq}$ or $0.25 \mathrm{mCi} / \mathrm{kg}$ initially, infused at a constant speed over $30 \mathrm{sec}$.). Data acquisition (correcting for random decay and electronic deadtime only) commenced for $90 \mathrm{sec}$. upon arrival of activity into the head as evidenced by consistently rising true counts. Each experimental scan contained data from one type of gamble, e.g., AG vs. RG (i.e., block design). Interscan interval was about 10 minutes. Images were reconstructed by filtered backprojection including nonorthogonal angles to a final image resolution of $10 \mathrm{~mm}$ full-width at halfmaximum.

\section{Analysis}

Image analysis was performed using software provided by Minoshima (Minoshima et al. 1993, Minoshima et al. 1994). Data were normalized to whole-brain average blood flow; image fiducials were used to localize the intercommissural plane; images were coregistered; anatomical standardization followed nonlinear transformation into a standard stereotaxic space (Talairach and Tournoux 1988). Voxel-by-voxel statistics (z-scores) were computed as the difference in condition means divided by the adjusted pooled standard deviation.

A threshold of $z=3.3$ reflects a significance level of 0.001 , which corrects for multiple comparisons. Several standard atlases were use to determine anatomical designations and approximate Brodmann areas (Talairach and Tournoux 1988, Schmahmann et al. 1997, Öngur and Price 2000).

\section{Results}

The behavioral data (choices made) in the four experimental conditions are summarized in Figure 2. The graph shows the difference between the proportions
Figure 2 The Interaction in Choice Behavior Between Knowledge Structure (Risk, Ambiguity) and Payoff Structure (Gains, Losses)

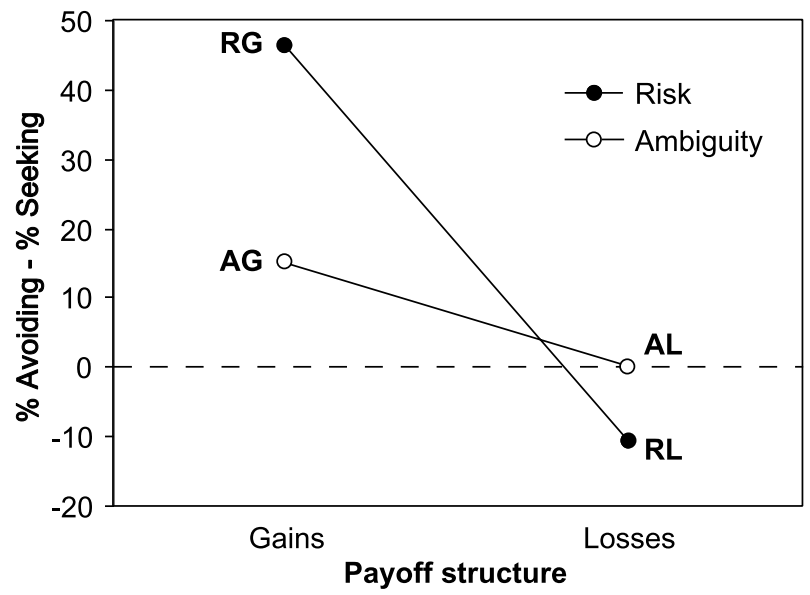

Note. The plotted points indicate the percentage difference between risk (ambiguity)-avoiding and risk (ambiguity)-seeking choices in the four conditions. The behavioral difference is greater under risk $(60 \%)$ than ambiguity $(15 \%)$ and the sign of the difference changes for losses but not for gains. Because the distance between $R G$ and $R L$ is much greater than the distance between $A G$ and $A L$, we predict that we will be able to uncover significant brain activation in examining the contrast between subtraction $R G-R L$ and subtraction $A G-A L$.

of ambiguity-avoiding and ambiguity-seeking choices in AG and AL conditions (15\% and $0 \%$, respectively) and the difference between the proportions of riskavoiding and risk-seeking choices in the RG and RL conditions ( $46 \%$ and $-11 \%$, respectively). Participants preferred ambiguity in neither gains nor losses. They also avoided the riskier (higher variance) gamble under gains, but sought the riskier gamble under losses. A within-subjects analysis of variance rejects the hypothesis that subjects' behavior is equivalent in all four conditions, $F(3,24)=5.87, p<0.005$. The observations shown in Figure 2 converge with the choice behavior in numerous experiments (Kahneman and Tversky 1979, Curley et al. 1986, Cohen et al. 1987, Tversky and Kahneman 1992, Tversky and Fox 1995).

The contrasts in brain activity between gains/losses and ambiguity/risk conditions, and their interaction, were calculated voxel-wise ${ }^{5}$ after anatomical

\footnotetext{
${ }^{5} \mathrm{~A}$ "voxel" is the volumetric equivalent of the more familiar "pixel." Here, a voxel represents a volume of brain in stereotactic space ( $2.25 \mathrm{~mm}$ on each side).
} 


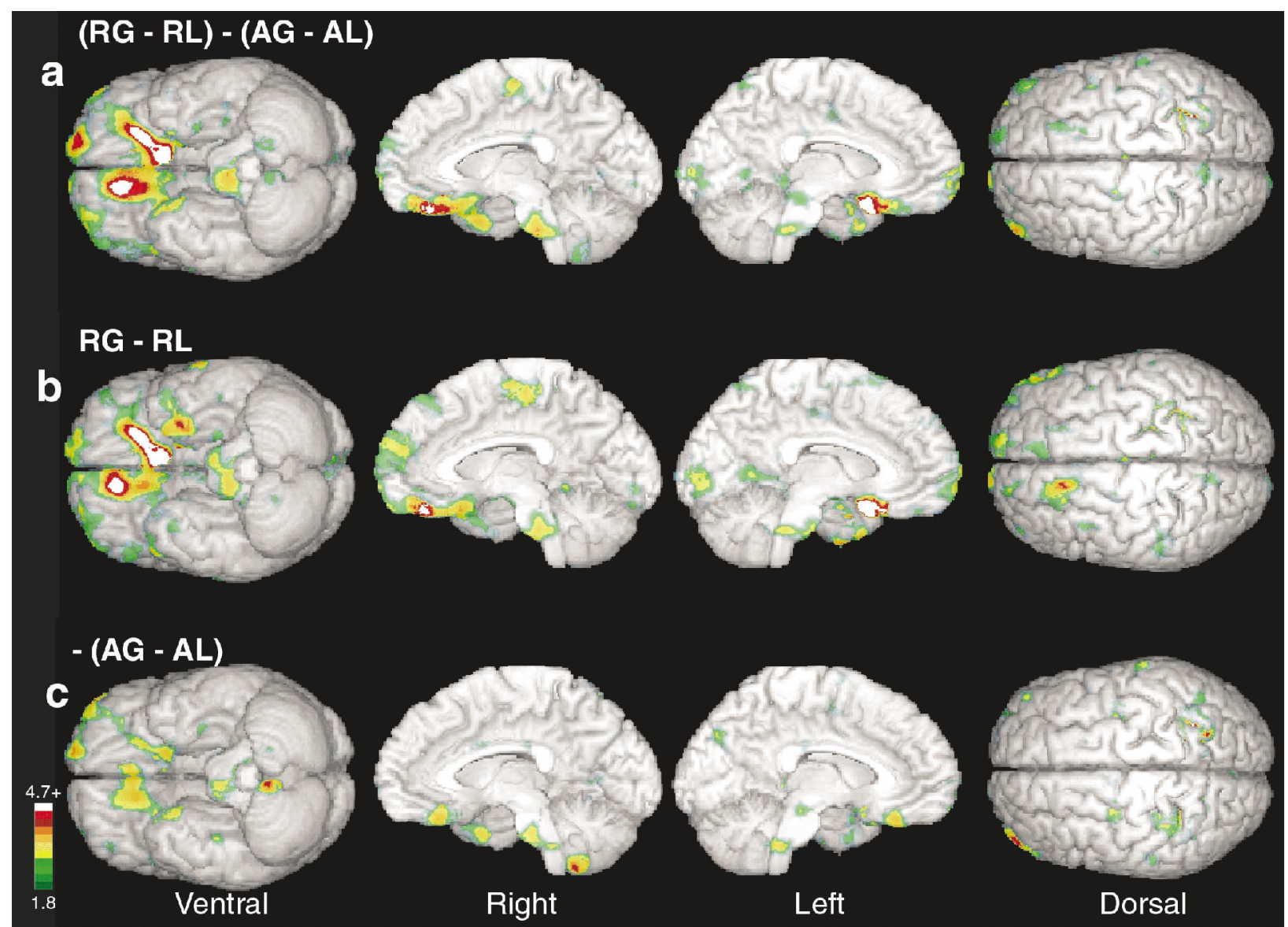

Note. (a) The top row shows brain locations differentially activated by the contrast between gain vs. loss under risk, compared to gain vs. loss under ambiguity, $[(R G-R L)-(A G-A L)$; Risk, R; ambiguity, A; gains, G; losses, L], revealing a ventromedial network. (b) The risk component is largely responsible for the interaction. (C) The ambiguity component plays a lesser role.

standardization across participants. In Figures 3 and 4, we show those voxels in both tails of the distribution. A standard magnetic resonance image (MRI), warped into stereotactic space, shows the structure of the brain in gray scale. The color scale indicates the relative amount of regional cerebral blood flow in terms of $z$-score: the voxels that demonstrate the highest relative increase in blood flow from one condition (the subtrahend) to the other (the minuend) are coded in white (z-score $>4.7)$; $z$-scores lower than 1.8 are not displayed.

The pattern of rCBF enlisted by the interaction between belief structure (ambiguity or risk) and payoff structure (gains or losses) is shown in Figures 3, Row A, and 4, Row A. This interaction was computed as the voxel-wise difference in brain activity during choice behavior across all four conditions expressed as a compound difference between (1) ambiguity and risk, and (2) losses and gains, i.e., [(RG - RL) $(A G-A L)$ ]. Figure 3, Row A, shows the activation of a ventromedial network associated with the gainloss difference in risky gambles that is not present in the gains-loss difference under ambiguity. Figure 4, Row A, shows the activation of a dorsomedial network with a loss-gain difference in risky gambles that is not present in the loss-gain difference under ambiguity. Figures 3, Row B, and 4, Row B, show the risk component of the interaction separately [i.e., (RG RL)]. Figures 3, Row C, and 4, Row C, show the ambiguity component separately (i.e., $(\mathrm{AG}-\mathrm{AL})$ ). 
Figure 4 Neural Systems Activated During Processing in the Interaction $[(R L-R G)-(A L-A G)$, i.e., the Deactivation Corresponding to Figure 3

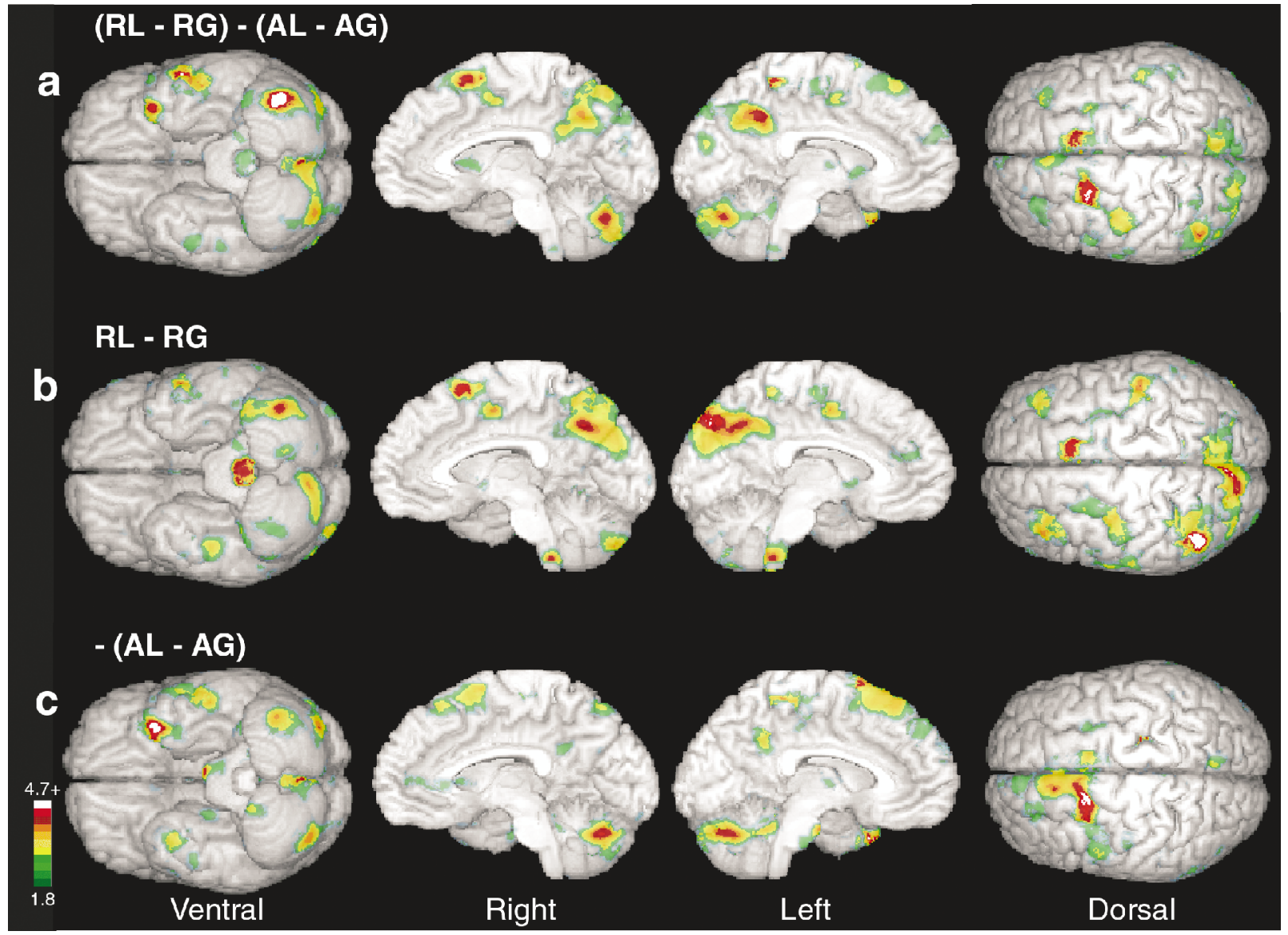

Note. (a) The top row shows brain locations differentially activated by the contrast between loss vs. gain under risk, compared to loss vs. gain under ambiguity, $[(R L-R G)-(A L-A G)$; Risk, R; ambiguity, A; gains, G; losses, L], revealing a dorsomedial network. (b) The risk component is largely responsible for the interaction. (C) The ambiguity component plays a lesser role.

The behavioral graph aids us in interpreting the brain phenomena. We note that the difference in observed behavior between AL and AG is small (0.15, see Figure 2) while the difference between RL and RG is relatively large (0.57). Examination of Figure 3 reveals differential ventromedial activation consistent with observed differences in behavioral data: most of the interaction effect is driven by the difference in risk conditions (Figure 3, Row B), with very little contribution by the difference in ambiguity conditions (Figure 3, Row C). Similarly, most of the interaction seen in dorsomedial cortex (Figure 4, Row A) is driven by the difference in the risk conditions (Figure 4, Row B), with very little contribution by the difference in ambiguity conditions (Figure 4, Row C).
The pattern of activation and deactivation seen in Figures 3 and 4 is the neural counterpart of the behavioral interaction seen in Figure 2. This study is among the first of its kind to identify the patterns of neuronal activity that support observed human choice behavior.

Tables 1 and 2 display the brain regions, approximate Brodmann areas, locations (in Talairach stereotactic coordinates), and significance levels of voxels isolated by the interaction between belief structure and payoff structure. The robust ventromedial network (in the medial portion of the base of the brain), highlighted in Figure 3, Row A, includes phylogenetically older cortices (Table 1 ). These regions tie 
Table 1 Regions, Locations, and Magnitudes (Expressed As Zscores $>3.3$ ) in the Interaction Between Beliefs (Risk, R; Ambiguity, A) and Payoffs (Gains, G; Losses, L): [(RG-RL)$(A G-A L)]$

\begin{tabular}{lccrrc}
\hline Brain Region & Brodmann Area & $X$ & $Y$ & $Z$ & $Z$-score \\
\hline Orbitofrontal & $13 \mathrm{~b}$ & -17 & 23 & -16 & 5.2 \\
Gyrus rectus & $14 \mathrm{c}$ & -8 & 10 & -18 & 4.9 \\
Medial orbitofrontal & $10 \mathrm{~m}, \mathrm{r}$ & 12 & 37 & -20 & 4.8 \\
Intraparietal sulcus & 7 & 24 & -51 & 52 & 4.7 \\
Frontal pole* & $10 \mathrm{p}$ & -15 & 64 & -11 & 4.4 \\
Brainstem, pons & & 6 & -28 & -32 & 3.7 \\
Frontal pole & $10 \mathrm{p}$ & -44 & 55 & 9 & 3.6 \\
Inferior frontal gyrus & $47 / 12 \mathrm{~m}$ & 33 & 19 & -14 & 3.6 \\
Inferior frontal gyrus & $47 / 12 \mathrm{~m}, \mathrm{I}$ & 26 & 12 & -20 & 3.4 \\
Entorhinal cortex & 28 & 19 & 5 & -27 & 3.4 \\
Parietal lobe & $7 / 40$ & 26 & -40 & 52 & 3.3 \\
\hline
\end{tabular}

* Asterisk indicates a focus on the edge of the brain that could be artifactual. Caution is appropriate when considering this region.

into structures such as the amygdala and hypothalamus that process emotion and the internal milieu. Clinical pathologic and neuropsychological studies have already suggested the critical role of the orbital gyrus and ventromedial prefrontal cortices in human emotion, decision making, and evaluation of outcomes (Öngur and Price 2000, Tversky and Fox 1995, Tversky and Kahneman 1992, Breiter et al. 2001, Damasio 1999). The dorsomedial network (at the top of the head near the midline), highlighted in Figure 4, Row A, contains phylogenetically newer cortices that may represent specialization for processing of risk in decision making, Table 2. An extensive literature supports that these regions can reflect components relevant to visual and spatial representation (e.g., precuneus, parietal lobe), to calculation procedures (e.g., parietal lobe, cerebellum), and to executive processes (e.g., dorsal prefrontal cortex). Thus are consistent with the interpretation that the RL condition generates more calculations and comparisons when contrasted with RG, AG, and AL. More comparisons divert activation away from the ventromedial area.

\section{Discussion}

This study is concerned with choices between gambles which payoff in dollars, the fundamental concern of experimental economics and a salient topic in
Table 2 Regions, Locations, and Magnitudes (Expressed As Zscores $>3.3$ ) in the Interaction Between Beliefs (Risk, R; Ambiguity, A) and Payoffs (Gains, G; Losses, L): [(RL - RG) $(\mathrm{AL}-\mathrm{AG})]$

\begin{tabular}{lcrrrc}
\hline Brain Region & Brodmann Area & $\mathrm{X}$ & $\mathrm{Y}$ & $\mathrm{Z}$ & $\mathrm{Z}$-score \\
\hline Cerebellum VIIB & & -35 & -58 & -45 & 5.1 \\
Middle temporal gyrus & 21 & -48 & -1 & -20 & 4.5 \\
Superior frontal gyrus & 6 & -21 & 5 & 56 & 4.4 \\
Paracentral lobule & 5 & -6 & -40 & 54 & 4.4 \\
Temporal pole* & 38 & -33 & 17 & -32 & 4.3 \\
Pre-SMA & 6 & 6 & 12 & 56 & 4.1 \\
Cerebellar Vermis VI & & 3 & -69 & -27 & 3.9 \\
Precuneus & 7 & -6 & -49 & 32 & 3.9 \\
Inferior parietal lobe & $39 / 40$ & -44 & -60 & 43 & 3.8 \\
Precuneus & $7 / 31$ & -1 & -55 & 36 & 3.6 \\
Cerebellum Crus I & & 30 & -76 & -34 & 3.5 \\
\hline
\end{tabular}

* Asterisk indicates a focus on the edge of the brain that could be artifactual. Caution is appropriate when considering this region.

decision making. Our analyses indicate that the interaction between belief structure (ambiguity/risk) and payoff structure (gain/loss) shapes the distribution of brain activity during choice. A simple manipulation of belief structure and payoff structure produces focal activations and deactivations that suggest two disparate, but functionally integrated, choice systems with sensitivity to loss: a neocortical dorsomedial system related to loss processing when evaluating risky gambles, and a more primitive ventromedial system related to processing of other stimuli. This anatomy suggests that choice under loss generates more use of the calculational part of the brain and diminishes the role of visceral representations in the ventromedial system which appear to be present under the other conditions, risk gain, ambiguity gain and ambiguity loss. The ventromedial system arose phylogenetically earlier and likely supports decision making in animals without more developed neocortices.

The present findings complement and extend the recent findings of Breiter et al. (2001), who mapped hemodynamic responses to the expectation and experience of monetary rewards and losses. Except for some overlap in frontal poles and orbital cortex, the neural regions are strikingly different between these two studies as might be expected given the differences in experimental design. We focused upon isolating brain regions critical in the choice process per se, 
while Breiter et al. specifically excluded the choice process in their design. Our subjects did not experience feedback about outcomes; they knew that their outcome would be determined at the end of the study by a random draw from their past choices. Finally, the contribution of expectancy is minimized in the present study through its absence in the ambiguity condition as well as cancellation in the interaction effect.

These two studies highlight distinct processes and anatomy relevant to decision making. It appears from Breiter et al. (2001) that structures relevant to expectancy and the experience of outcomes are ventral brain structures including bilateral orbitofrontal cortices (with more anterior predominance); amygdala; sublenticular extended amygdala; nucleus accumbens; hypothalamus; and ventral tegmentum. In contrast, systems most closely tied to the choice process per se are medial with ventral vs. dorsal distribution depending upon the knowledge structure and payoff structure. The ventromedial orbital regions associated with ambiguity processing under loss show a more posterior distribution than those related to the processing of prospects.

In both economics and decision theory, it is generally assumed that belief structure and payoff structure are separable, if not independent. This study shows that they can interact to reveal two dissociable choice systems.

\section{Acknowledgments}

The authors thank their volunteer participants; Joel T. Lee, Patricia J. Pardo, Jennifer C. Nagode, and other colleagues in the Cognitive Neuroimaging Unit; and the technical staff of the PET Imaging Service of the Minneapolis VA Medical Center. This work was supported by the National Science Foundation and the Department of Veterans Affairs.

\section{References}

Breiter, H. C., I. Aharon, D. Kahneman, A. Dale, P. Shizgal. 2001. Functional imaging of neural responses to expectancy and experience of monetary gains and losses. Neuron 30 619-639.

— J. D. Berke, W. A. Kennedy, B. R. Rosen, S. E. Hyman. 1996. Activation of striatum and amygdala during reward conditioning: An fMRI study. Neuroimage 3 S220.
Camerer, C., M. Weber. 1992. Recent developments in modeling preferences: Uncertainty and ambiguity. J. Risk Uncertainty 5 325-370.

Cohen, M., J. Jaffray, T. Said. 1987. Experimental comparisons of individual behavior under risk and under uncertainty for gains and for losses. Organ. Behavior Human Decision Process. 39 $1-22$.

Curley, S. P., J. F. Yates. 1985. The center and range of the probability interval as factors affecting ambiguity preferences. Organ. Behavior Human Decision Process. 36 273-287.

—, R. A. Abrams. 1986. Psychological sources of ambiguity avoidance. Organ. Behavior Human Decision Process. 38 230-256.

Damasio, A. R. 1999. The Feeling of What Happens: Body and Emotion in the Making of Consciousness. Harcourt Brace \& Co., New York.

Ellsberg, D. 1961. Risk, ambiguity, and the Savage axioms. QJE 75 643-669.

Gilboa, I., D. Schmeidler. 1985. MaxMin utility with non-unique prior. J. Monet Econom. 18 141-153.

Heath, C., A. Tversky. 1991. Preference and belief: Ambiguity and competence in choice under uncertainty. J. Risk Uncertainty 4 $5-28$.

Howard, R. A. 1992. The cogency of decision analysis. W. Edwards, ed. Utility: Theories, Measurement, Applications. Kluwer Academic Publishers, Dordrecht, The Netherlands.

Kahneman, D., A. Tversky. 1979. Prospect theory: An analysis of decision under risk. Econometrica 47 263-291.

Knight, F. H. 1921. Risk, Uncertainty, and Profit. Houghton Mifflin, New York.

Minoshima, S., R. A. Koeppe, K. A. Frey, D. E. Kuhl. 1994. Anatomic standardization: Linear scaling and nonlinear warping of functional brain images. J. Nucl. Med. 35 1528-1537.

Minoshima, S., R. A. Koeppe, M. A. Mintun, K. L. Berger, S. F. Taylor, K. A. Frey, D. E. Kuhl. 1993. Automated detection of the intercommissural line for stereotactic localization of functional brain images. J. Nucl. Med. 34 322-329.

Öngur, D., J. L. Price. 2000. The organization of networks within the orbital and medial prefrontal cortex of rats, monkeys and humans. Cereb. Cortex. 10 206-219.

Silbersweig, D., E. Stern, C. Frith, C. Cahill, L. Schnorr, S. Grootoonk. 1993. Detection of thirty-second cognitive activations in single subjects with positron emission tomography: A new low-dose $\mathrm{H} 215 \mathrm{O}$ Regional Cerebral blood flow threedimensional imaging technique. J. Cereb. Blood Flow Metab. 13 617-629.

Talairach, J., P. Tournoux. 1988. Co-Planar Stereotaxic Atlas of the Human Brain. Thieme, New York.

Tversky, A., C. R. Fox. 1995. Weighing risk and uncertainty. Psych. Rev. 102 269-283.

— D. Kahneman. 1992. Advances in prospect theory: Cumulative representation of uncertainty. J. Risk Uncertainty 5323.

Worsley, K. J., J. B. Poline, A. C. Vandal, K. J. Friston. 1995. Tests for distributed, nonfocal brain activations. Neuroimage 2 183-194. 